\title{
Ein sensitiver Detektor für die Gaschromatographie mittels Ionisation durch eine dielektrische Barrierenentladung
}

\author{
Ansgar T. Kirk, Torben Last, Jens Langejuergen, Stefan Zimmermann \\ Institut für Grundlagen der Elektrotechnik und Messtechnik, Fachgebiet Sensorik und Messtechnik, \\ Leibniz Universität Hannover, Appelstr. 9A, 30167 Hannover, Deutschland \\ kirk@geml.uni-hannover.de
}

\begin{abstract}
:
Ionenmobilitätsspektrometer (IMS) sind schnelle, hochsensitive Detektoren für Spurengase und bieten dank der typischerweise genutzten chemischen Gasphasenionisation Nachweisgrenzen im unteren ppt $_{v}$-Bereich für Messdauern von unter einer Sekunde. Dies macht sie nicht nur als stand-alone Geräte interessant, sondern hat unter anderem auch zu ihrer Nutzung als Detektoren für die Gaschromatographie geführt. Hierbei können sowohl positive Ionen als Ersatz für beispielsweise einen Photoionisationsdetektor als auch negative Ionen als Ersatz für beispielsweise einen Elektroneneinfangdetektor gemessen werden. Da der instrumentelle Aufwand eines vollständigen Ionenmobilitätsspektrometers im Gegensatz zu anderen Detektoren jedoch vergleichsweise hoch ist, wurde im Rahmen dieser Arbeit ein kompakter Detektor entwickelt, welcher ebenfalls eine chemische Gasphasenionisation nutzt. Dieser besitzt jedoch im Gegensatz zu einem lonenmobilitätsspektrometer keine eigene Trenndimension für verschiedene Substanzen und ist daher wesentlich simpler und kostengünstiger aufzubauen. Ein aktueller Demonstrator erreicht dabei bereits eine Nachweisgrenze von $4 \mathrm{ppb}_{v}$ Aceton in gereinigter Luft für einen Analytgasfluss von $100 \mathrm{mls} / \mathrm{min}$.
\end{abstract}

Keywords: Gaschromatographie, Detektor, lonenmobilitätsspektrometrie, dielektrische Barrierenentladung

\section{Einleitung}

Ionenmobilitätsspektrometer (IMS) trennen und identifizieren Stoffe anhand der Bewegung ihrer Ionen durch ein Neutralgas unter dem Einfluss eines elektrischen Feldes [1]. Eine Messung mit einem solchen Gerät dauert nur wenige Millisekunden, wobei für eine Mittelungsdauer von unter einer Sekunde Nachweisgrenzen im unteren $\mathrm{ppt}_{\mathrm{v}}$-Bereich erzielt werden können [2]. Aufgrund dieser hervorragenden Sensitivität bei kurzer Messdauer werden Ionenmobilitätsspektrometer nicht nur als stand-alone Geräte genutzt, sondern beispielsweise auch als Detektoren für die Gaschromatographie (GC). Bereits das erste als analytisches Instrument eingesetzte lonenmobilitätsspektrometer [3], damals noch als Plasmachromatograph bezeichnet, wurde als Detektor an einen Gaschromatographen gekoppelt. Da die Messdauer des Ionenmobilitätsspektrometers um Größenordnungen unter der des Gaschromatographen liegt, kann jeder Peak im Chromatogramm nochmals vollständig im lonenmobilitätsspektrometer analysiert werden.

Das elektrische Feld im lonenmobilitätsspektrometer kann hierbei invertiert werden, um entweder positive oder negative lonen untersu- chen zu können. Bei der Messung im positiven Modus werden dabei Stoffe detektiert, welche üblicherweise mit Photo- oder Flammionisationsdetektoren gemessen werden. Bei der Messung im negativen Modus werden hingegen Stoffe detektiert, welche üblicherweise mit Elektroneneinfangdetektoren gemessen werden. Der entscheidende Unterschied ist hierbei, dass die drei genannten lonisationsdetektoren direkt bestimmte Stoffgruppen ionisieren. Der hieraus resultierende lonenstrom ist dann ein Maß für die in der Probe vorhandene Stoffmenge. Ionenmobilitätsspektrometer könnten die so erzeugten Ionen analysieren, um so Aufschluss über den tatsächlich vorhandenen Stoff zu geben [4]. Typischerweise nutzen Ionenmobilitätsspektrometer jedoch keine direkte lonisation, sondern eine chemische Gasphasenionisation bei Atmosphärendruck (engl. Atmospheric Pressure Chemical Ionization, APCl). Bei dieser wird zunächst eine große Zahl sogenannter Reaktantionen gebildet, welche dann über eine weiche chemische Reaktion die zu analysierenden Moleküle ionisieren. Da hierbei mit einer großen Anzahl langlebiger Reaktantionen selbst kleinste Stoffmengen effizient und effektiv ionisiert werden können, stellt die chemische Gasphasenionisation den Schlüssel zur hervor- 
ragenden Sensitivität von Ionenmobilitätsspektrometern dar. Darüber hinaus stellt sie auch eine gewisse Selektivität zur Verfügung, da typischerweise vorhandene Hintergrundgase der Luft wie Stickstoff, Sauerstoff, Kohlenstoffdioxid oder Edelgase keinen Messeffekt verursachen. Dies liegt daran, dass ihre Ionisation gegenüber den entstehenden Reaktantionen energetisch ungünstiger wäre.

Trotz all dieser Vorteile besitzt die chemische Atmosphärendruckionisation einen entscheidenden Nachteil, der ihre Nutzbarkeit stark limitiert. Da ihr Messeffekt nur die Umwandlung einer lonenspezies in eine andere darstellt, bleibt der von ihr erzeugte lonenstrom unabhängig von den vorhandenen Spurengasen nahezu konstant. Daher ist keine einfache Auswertung des lonenstroms wie bei der Photo- oder Flammionisation möglich, sondern es wird immer ein ionenselektiver Detektor benötigt, typischerweise ein lonenmobilitäts- oder Massenspektrometer. Für Anwendungen, bei denen der Gaschromatograph die benötigte Trennleistung zur Verfügung stellen kann, und somit die zusätzliche Trennleistung eines solchen Detektors außer zur Nutzung der chemischen Gasphasenionisation nicht benötigt wird, stellt ein lonenmobilitätsspektrometer und erst recht ein Massenspektrometer jedoch einen absolut unverhältnismäßigen instrumentellen Aufwand dar. Für diese Anwendungen ist daher ein alternatives Konzept von Interesse. Dieses sollte die für die Gaschromatographie interessanten Eigenschaften eines Ionenmobilitätsspektrometers, zum einen die hochsensitive chemische Gasphasenionisation und zum anderen die Fähigkeit, Analyt- von Reaktantionen zu trennen, mit einem deutlich simpleren und kostengünstigeren Aufbau vereinen.

\section{Detektorkonzept}

Das grundlegende Konzept eines solchen Detektors ist in Abb. 1 dargestellt. Er basiert auf dem bekannten Konzept eines sogenannten Aspiration Condenser Ionenmobilitätsspektrometers [5], welches Ionen mit einem Gasstrom transportiert und diese gleichzeitig mit einem orthogonalen elektrischen Feld ablenkt. Da nur die Bewegung in Richtung des elektrischen Feldes von der Mobilität der Ionen abhängt, können diese so getrennt werden. Im vorliegenden Anwendungsfall ist nur eine Trennung zwischen Reaktantionen und anderen lonen notwendig, sodass lediglich zwei Detektorelektroden und eine konstante Driftspannung genutzt werden. Diese ist so gewählt, dass ein nur aus Reaktantionen bestehender Ionenstrom sich gleichmäßig auf beide Detektorplatten aufteilt und somit am Ausgang kein Differenzstrom gemessen wird. Analytionen besitzen hingegen eine andere Mobilität, werden folglich abgelenkt und verursachen einen messbaren Differenzstrom.

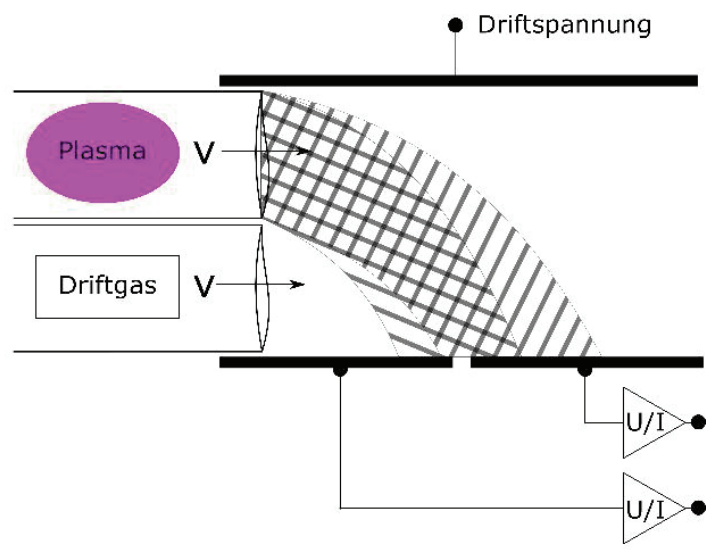

Abb. 1: Prinzip der Trennung von Reaktantionen (V) und Analytionen (/) im entwickelten lonendetektor.

Dieser wird über der Zeit aufgetragen, um das Chromatogramm zu erhalten.

Im vorliegenden Aufbau wird darüber hinaus das sogenannte lon Focussing [5] verwendet, bei dem die lonen nur im oberen Bereich des Detektors eingeleitet werden. Dies erhöht die Trennleistung eines Aspiration Condenser Ionenmobilitätsspektrometers erheblich, da die Ionen einen definierten Startpunkt aufweisen und so ihr Endpunkt auf den Detektorelektroden primär von ihrer lonenmobilität und diffusiven Prozessen bestimmt wird. Dabei verkürzt eine höhere Strömungsgeschwindigkeit des Gasflusses die Aufenthaltsdauer im Detektor und verbessert somit die Trennleistung aufgrund der reduzierten Diffusion. Dies gilt jedoch nicht unbegrenzt, da zum einen die Qualität des Ion Focussing ebenfalls limitierend wirken kann und zum anderen für hohe Durchflüsse keine rein laminare Strömung mehr angenommen werden kann. 


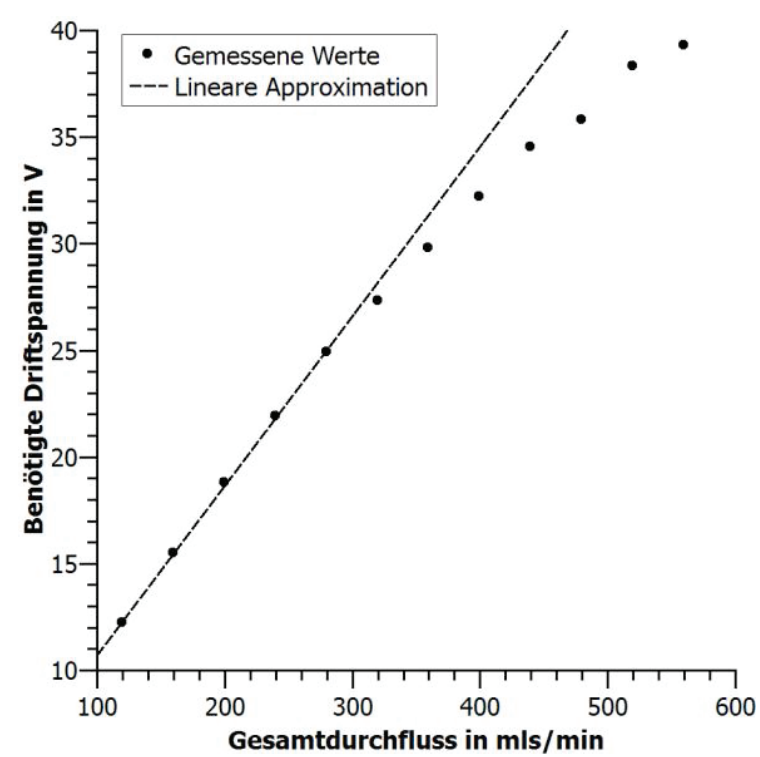

Abb. 2: Benötigte Driftspannung als Funktion des verwendeten Gasflusses.

Für eine laminare Strömung sollte die Strömungsgeschwindigkeit linear mit dem Volumenstrom zunehmen und damit auch linear eine höhere Driftspannung benötigt werden, damit die Reaktantionen in der Mitte zwischen den Detektorplatten auftreffen. Um dies zu untersuchen, ist in Abb. 2 die benötigte Driftspannung über dem genutzten Volumenstrom dargestellt. Dabei ist zu erkennen, dass diese zunächst wie erwartet linear ansteigt, für Flüsse über $300 \mathrm{mls} / \mathrm{min}$ jedoch zunehmend abflacht. Aufgrund der somit zu erwartenden Nichtidealitäten wurde in den folgenden Studien hauptsächlich der Bereich von Gesamtflüssen unter $300 \mathrm{mls} / \mathrm{min}$ betrachtet.

\section{Dielektrische Barrierenentladung}

Zusätzlich zu dem Detektor zur Trennung von Reaktant- und Analytionen wird eine Ionenquelle benötigt, welche die Reaktantionen für die chemische Gasphasenionisation bereitstellt. Darüber hinaus ist aufgrund des oben beschriebenen Ion Focussing eine lonenquelle von Interesse, welche lokal begrenzt lonen erzeugt. Hierzu wurde in dieser Arbeit eine lonenquelle auf Basis einer dielektrischen Barrierenentladung [6] entwickelt, welche mittels zweier planarer Elektroden ein Plasma direkt in einer Kapillare zünden und somit die Analyte ionisieren kann. Da hierzu nur eine kleine, räumlich stark begrenzte Entladung notwendig ist, benötigt der aktuelle Demonstrator lediglich eine elektrische Leistung von $1 \mathrm{~W}$. Daher finden sowohl das für die Entladung benötigte Hochspannungsnetzteil als auch die Elektroden auf der in Abb. 3 gezeigten, $60 \mathrm{~mm}$ mal $70 \mathrm{~mm}$ großen Platine Platz.
Die durch die dielektrische Barrierenentladung erzeugten lonen werden dann durch den Gasfluss in der oberen Kapillare aus dem Entladungsbereich in den Detektor transportiert.

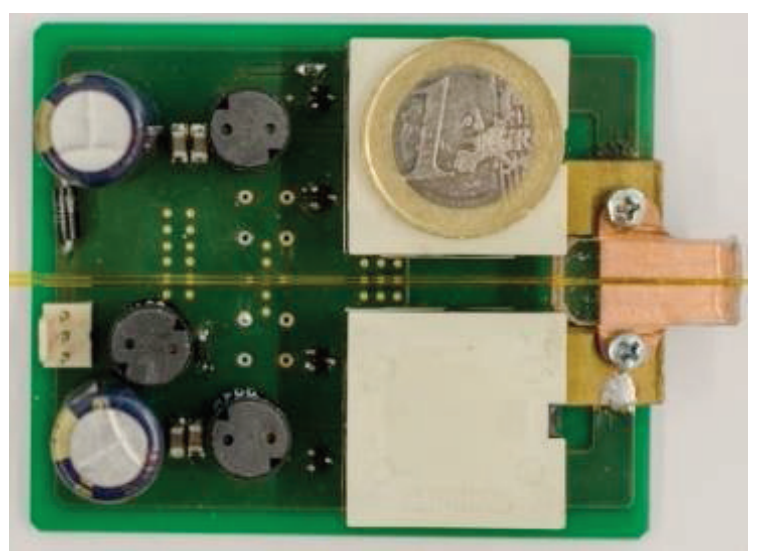

Abb. 3: Platine mit Hochspannungsnetzteil, Zuleitungskapillaren sowie den Elektroden für die dielektrische Barrierenentladung.

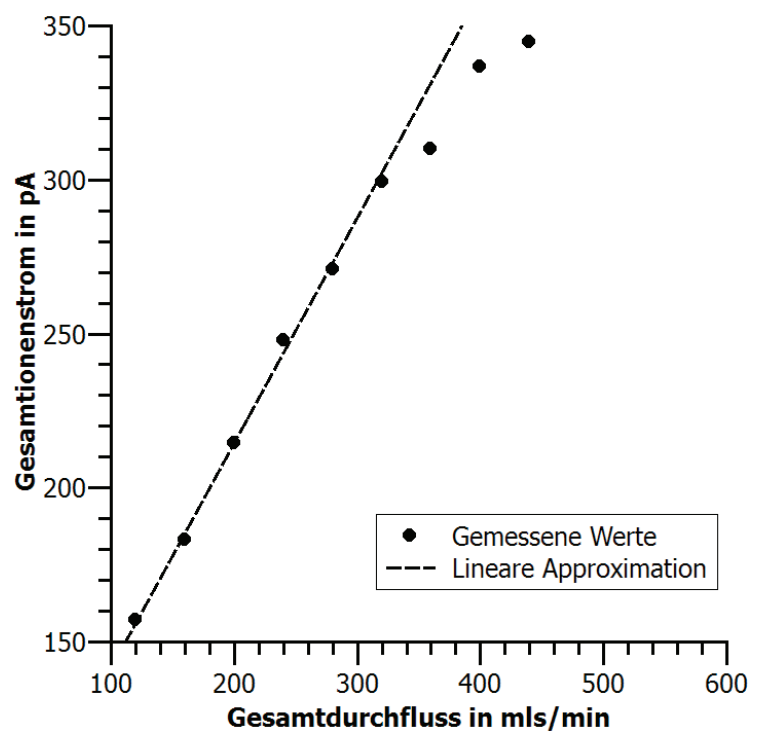

Abb. 4: Gesamtionenstrom als Funktion des verwendeten Gasflusses.

Daher sorgt ein höherer Gasfluss nicht nur für eine bessere Trennleistung im Detektor, sondern wie in Abb. 4 gezeigt auch für einen höheren Gesamtionenstrom. Da das Rauschen dabei nicht zunimmt, ist für höhere Flüsse eine Verbesserung der Nachweisgrenzen zu erwarten.

\section{Messungen}

Im Folgenden wird eine erste Charakterisierung des entwickelten Detektors vorgestellt. Um ein gleichmäßiges Strömungsprofil über den Querschnitt des Detektors zu erhalten, wird durch beide der in Abb. 1 gezeigten Kapillaren derselbe Volumenstrom geleitet. Über die obere Kapillare wird dabei das Analytgas zugeführt, 
während die untere Kapillare mit gereinigter Luft durchspült wird. Beide Flüsse sind für alle folgenden Experimente auf $100 \mathrm{mls} / \mathrm{min}$ gestellt, sodass sich ein Gesamtdurchfluss von $200 \mathrm{mls} / \mathrm{min}$ ergibt. Dieser liegt gemäß der Messungen in Abb. 2 und Abb. 4 innerhalb des Bereichs, indem ein lineares Verhalten der abhängigen Größen zu erwarten ist.

Abb. 5 zeigt den gemessenen Differenzstrom, wenn durch die obere Kapillare ein Gemisch aus gereinigter Luft und Aceton in verschiedenen Konzentrationen gegeben wird. Aus der Messung ergibt sich eine Sensitivität von $260 \mathrm{fA} / \mathrm{ppb}_{\mathrm{v}}$ für Aceton, wobei die Standardabweichung des Rauschens 350 fA beträgt. Hieraus lässt sich für Aceton eine Nachweisgrenze von $4 \mathrm{ppb}_{\mathrm{v}}$ ermitteln. Dies ist bereits eine erhebliche Verbesserung gegenüber dem ersten Demonstrator, welcher eine Nachweisgrenze von $150 \mathrm{ppb}_{\mathrm{v}}$ Aceton bei $200 \mathrm{mls} / \mathrm{min}$ Analytgasfluss beziehungsweise $400 \mathrm{mls} / \mathrm{min} \mathrm{Ge}$ samtfluss erreichte.

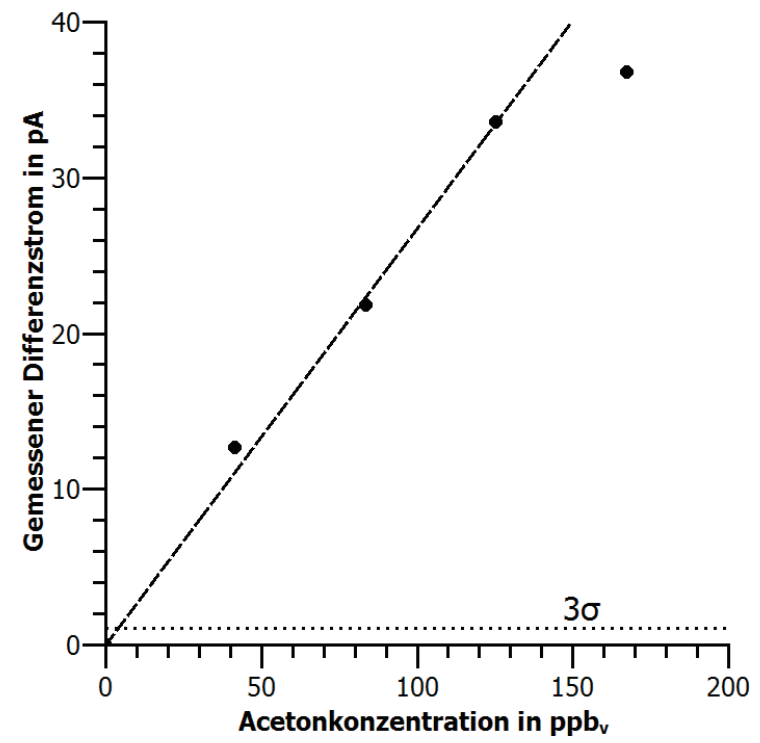

Abb. 5: Gemessener Differenzstrom als Funktion der Acetonkonzentration im Analytgas

Es ist anzumerken, dass das Rauschen des Messsignals primär durch hochfrequente Schwankungen der dielektrischen Barrierenentladung selbst verursacht wird. Mit einer hierhingehend optimierten lonenquelle wäre daher nochmals eine deutliche Verbesserung der Nachweisgrenzen zu erwarten.

Anschließend wurde der Detektor an einen simplen, selbstkonstruierten Gaschromatographen gekoppelt. Dieser nutzt eine isotherm betriebene Säule mit $10 \mathrm{~m}$ Länge und einen Trägergasfluss von $10 \mathrm{mls} / \mathrm{min}$ gereinigter Luft. Da der Detektor jedoch idealerweise mit einem Analytgasfluss von $100 \mathrm{mls} / \mathrm{min}$ betrieben werden sollte, ist die Zugabe von Make-Up-Gas notwendig.

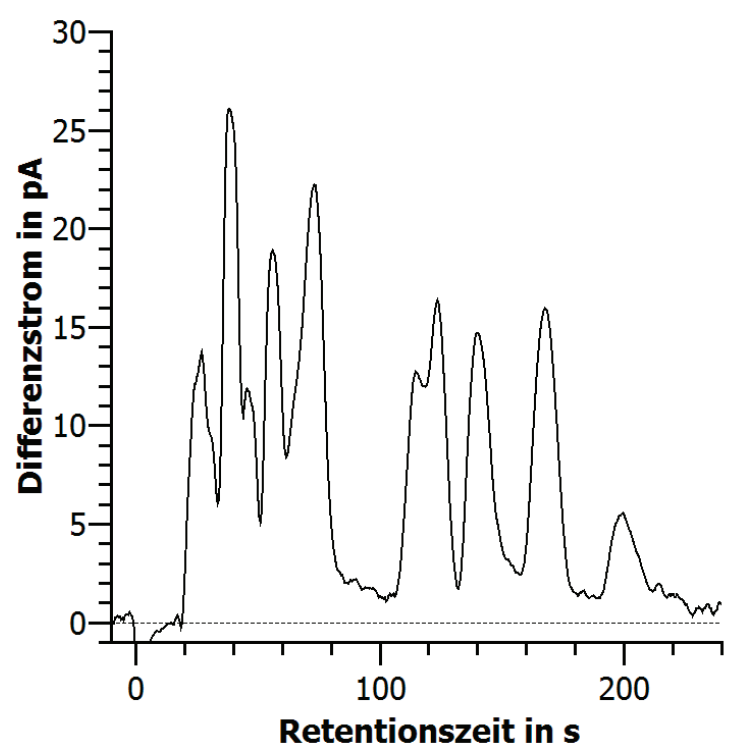

Abb. 6: Chromatogramm einer Kaffeeprobe

Durch diese Verdünnung um den Faktor 10 verschlechtern sich daher in diesem Aufbau die Nachweisgrenzen entsprechend gegenüber den des Detektors alleine. Dennoch ist, wie am in Abb. 6 dargestellten Chromatogramm einer Kaffeeprobe erkennbar, die Nutzung des Detektors in dieser Anwendung bereits jetzt problemlos möglich.

\section{Zusammenfassung}

In dieser Arbeit wurde zum ersten Mal ein kompakter Detektor für die Gaschromatographie auf Basis der Ionisation durch eine dielektrische Barrierenentladung vorgestellt. Der aktuelle Demonstrator erreicht dabei eine Nachweisgrenze von $4 \mathrm{ppb}_{\mathrm{v}}$ Aceton in gereinigter Luft. Da diese Nachweisgrenze jedoch noch hinter der von optimierten lonenmobilitätsspektrometern zurückbleibt, diese aber nach demselben Prinzip ionisieren, ist noch Optimierungspotential zu erwarten. Ansatzpunkte sind hier insbesondere die Verbesserung der dielektrischen Barrierenentladung hinsichtlich eines geringeren Rauschens sowie des Betriebs bei kleineren Gasflüssen. Ersteres würde direkt in die Nachweisgrenze eingehen, während letzteres das zurzeit noch notwendige Verdünnen der aus dem Gaschromatographen eluierenden Stoffe eliminieren würde.

\section{Quellen}

[1] G. Alan Eiceman, Z. Karpas, Hill Jr., Herbert H., Ion mobility spectrometry, (2014)

[2] A. T. Kirk, M. Allers, P. Cochems, J. Langejuergen, S. Zimmermann, A compact high resolution ion mobility spectrometer for fast trace gas analysis, 138, 5200-5207(2013); doi: 10.1039/c3an00231d 
[3] M. J. Cohen, F. W. Karasek, Plasma Chromatography --A New Dimension for Gas Chromatography and Mass Spectrometry, Journal of chromatographic science 8, 330-337(1970); doi: 10.1093/chromsci/8.6.330

[4] F. W. Karasek, D. M. Kane, Effect of oxygen on response of the electron-capture detector, Analytical Chemistry 45, 576-580(1973); doi: 10.1021/ac60325a016

[5] S. Zimmermann, N. Abel, W. Baether, S. Barth, An ion-focusing aspiration condenser as an ion mobility spectrometer, Sensors and Actuators B: Chemical 125, 428-434(2007); doi:

10.1016/j.snb.2007.02.038

[6] N. Na, M. Zhao, S. Zhang, C. Yang, X. Zhang, Development of a dielectric barrier discharge ion source for ambient mass spectrometry, Journal of the American Society for Mass Spectrometry 18, 1859-1862(2007); doi: 10.1016/j.jasms.2007.07.027 